\title{
Erratum
}

\section{Associations of parental bonding and adolescent internet addiction symptoms with depression and anxiety in parents of adolescents with attention deficit/hyperactivity disorder}

CHIH-KaI Wong ${ }^{1}$

https://orcid.org/0000-0003-3209-8930

Yu-Min CHEN ${ }^{2}$

https://orcid.org/0000-0002-4886-3783

Cheng-FANG Yen2,3

https://orcid.org/0000-0003-1156-4939

ISchool of Medicine, Chang Gung University, Taiwan, China.

2 Department of Psychiatry, Kaohsiung Medical University Hospital, Kaohsiung, Taiwan, China.

s Department of Psychiatry, School of Medicine, and Graduate Institute of Medicine, College of Medicine, Kaohsiung Medical University, Kaohsiung, Taiwan, China.

Received: 07/19/2018 - Accepted: 02/18/2019

DOl: 10.1590/0101-60830000000190

\section{Where it reads:}

1 School of Medicine, Chang Gung University, Taiwan, China.

2 Department of Psychiatry, Kaohsiung Medical University Hospital, Kaohsiung, Taiwan, China

${ }^{3}$ Department of Psychiatry, School of Medicine, and Graduate Institute of Medicine, College of Medicine, Kaohsiung Medical University, Kaohsiung, Taiwan, China.

It should read:

ISchool of Medicine, Chang Gung University, Taiwan.

2 Department of Psychiatry, Kaohsiung Medical University Hospital, Kaohsiung, Taiwan.

${ }^{3}$ Department of Psychiatry, School of Medicine, and Graduate Institute of Medicine, College of Medicine, Kaohsiung Medical University, Kaohsiung, Taiwan.

DOl: $10.1590 / 0101-60830000000207$ 